Vietnam Journal of Mechanics, NCST of Vietnam Vol. 25, 2003, No 1 (19-25)

\title{
THE POINCARÉ METHOD FOR A STRONGLY NONLINEAR DUFFING OSCILLATOR
}

\author{
NGUYen VAN DinH \\ Institute of Mechanics
}

It is well-known that the classical Poincaré method is limited to weakly nonlinear systems and for extending the range of validity of this method to strongly non - linear systems, various modifications have been developed.

Some authors have replaced the original parameter $\varepsilon$ by a new $\alpha$; the later is chosen such that the values $\alpha$ are always kept small regardless of the magnitude of $\varepsilon$. High degree of accuracy have been obtained. For instance in [2], for an undamped Duffing oscillator having large cubic non-linearity the free oscillation period evaluated by the " $\alpha$ method" in the fourth approximation (solution to $0\left(\alpha^{5}\right)$ ) is identical with that given by the exact solution, even for large $\varepsilon a^{2}(=1000)$.

In the present article, the mentioned Duffing oscillator is examined by another modified Poincaré method. Practically acceptable results are obtained.

$\S 1$. System under consideration. Period from the exact solution

Consider an oscillator governed by the differential equation

$$
\ddot{x}+x+\varepsilon x^{3}=0,
$$

where $x$ is an oscillatory variable; overdots denote differentiation with respect to time $t ; \varepsilon$ is the cubic non-linearity coefficient which is assumed to be positive and arbitrary ( $\varepsilon$ needs not to be small)

The attention is focused on the period of free oscillation satisfying initial conditions

$$
x(0)=a, \quad \dot{x}(0)=0 .
$$

The exact period is given by the formulae

$$
T_{e x}=\frac{4}{\sqrt{1+\varepsilon a^{2}}} \int_{0}^{\pi / 2} \frac{d \theta}{\sqrt{1-m \sin ^{2} \theta}}, \quad m=\frac{\varepsilon a^{2}}{2\left(1+\varepsilon a^{2}\right)}
$$

and the value $T_{e x}$ as function of $\varepsilon a^{2}$ was given in [2] (see the second column of the table) 
§2. Period from the standard Poincré method

For the sake of comparison, the standard Poincre method [1] is used first for the case of small $\varepsilon$.

Let $\omega$ be the frequency to be evaluated. Introducing the new time $\tau=\omega t$, the equation (1.1) can be written as:

$$
\omega^{2} x^{\prime \prime}+x+\varepsilon x^{3}=0,
$$

where primes denote differentiation with respect to $\tau$. According to the standard Poincaré method, both unknowns $x$ and $\omega$ are expanded in powers of $\varepsilon$, that is:

$$
\begin{aligned}
& x=x_{0}+\varepsilon x_{1}+\varepsilon^{2} x_{2}+\ldots \\
& \omega=1+\varepsilon \omega_{1}+\varepsilon^{2} \omega_{2}+\ldots
\end{aligned}
$$

and the initial conditions are replaced by the following ones

$$
\begin{array}{ll}
x_{0}(0)=a, & x_{0}^{\prime}(0)=0, \\
x_{i}(0)=0, & x_{i}^{\prime}(0)=0 .
\end{array}
$$

Substituting the expansions (2.2) and (2.3) into the equation (2.1) then equating coefficients of like powers of $\varepsilon$ yield a set of equation

$$
\begin{aligned}
x_{0}^{\prime \prime}+x_{0} & =0, \\
x_{1}^{\prime \prime}+x_{1} & =-2 \omega_{1} x_{0}^{\prime \prime}-\varepsilon x_{0}^{3}, \\
x_{2}^{\prime \prime}+x_{2} & =-2 \omega_{1} x_{1}^{\prime \prime}-\left(\omega_{1}^{2}+2 \omega_{2}\right) x_{0}^{\prime \prime}-3 x_{0}^{2} x_{1} .
\end{aligned}
$$

The general solution of the equation (2.6) satisfying the initial conditions (2.4) is

$$
x_{0}=a \cos \tau \text {. }
$$

Using (2.9), the equation (2.7) can be written in detailled form:

$$
\begin{aligned}
x_{1}^{\prime \prime}+x_{1} & =2 \omega_{1} a \cos \tau-\varepsilon a^{3} \cos ^{3} \tau \\
& =\left(2 \omega_{1}-\frac{3}{4} a^{2}\right) a \cos \tau-\frac{a^{3}}{4} \cos 3 \tau .
\end{aligned}
$$

To eliminate the secular term, $\omega_{1}$ should be taken as

$$
\omega_{1}=\frac{3 a^{2}}{8} .
$$

Then the solution $x_{1}$ satisfying the initial conditions $(2.5)$ is

$$
x_{1}=\frac{a^{3}}{32}(\cos 3 \tau-\cos \tau) .
$$


Going on this perturbation procedure, with regard to (2.9) - (1.12), we rewrite the equation (2.8) as:

$$
x_{2}^{\prime \prime}+x_{2}=\left(2 \omega_{2}+\frac{21 a^{4}}{128}\right) a \cos \tau+\frac{3 a^{5}}{16} \cos 3 \tau-\frac{3 a^{5}}{128} \cos 5 \tau .
$$

Eliminating the secular term gives:

$$
\omega_{2}=-\frac{21 a^{4}}{256}
$$

The solution $x_{2}$ satisfying the initial conditions $(2.5)$ is

$$
x_{2}=\frac{3 a^{5}}{128}(\cos \tau-\cos 3 \tau)+\frac{3 a^{5}}{3072}(\cos 5 \tau-\cos \tau) .
$$

Hence, respectively for the frequency $\omega$, the period $T$ and the solution $x$, we obtain

- in the first approximation

$$
\begin{aligned}
\omega^{(1)} & =1+\frac{3}{8} \varepsilon a^{2}, \\
T_{1} & =\frac{2 \pi}{\omega^{(1)}}=\frac{2 \pi}{1+\frac{3}{8} \varepsilon a^{2}}, \\
x^{(1)} & =a \cos \tau+\frac{\varepsilon a^{3}}{32}(\cos 3 \tau-\cos \tau) .
\end{aligned}
$$

- in the second approximation

$$
\begin{aligned}
\omega^{(2)}= & 1+\frac{3}{8} \varepsilon a^{2}-\frac{21}{256} \varepsilon^{2} a^{4}, \\
T_{2}= & \frac{2 \pi}{\omega^{(2)}}=\frac{2 \pi}{1+\frac{3}{8} \varepsilon a^{2}-\frac{21}{256} \varepsilon^{2} a^{4}}, \\
x^{(2)}= & a \cos \tau+\frac{\varepsilon a^{3}}{32}(\cos 3 \tau-\cos \tau)+\frac{3 \varepsilon^{2} a^{5}}{128}(\cos \tau-\cos 3 \tau) \\
& +\frac{3 \varepsilon^{2} a^{5}}{3072}(\cos 5 \tau-\cos \tau) .
\end{aligned}
$$

The values of $T_{1}, T_{2}$ as functions of $\varepsilon a^{2}$ are shown below in the third and fourth columns of the table.

We see that the results obtained from the standard Poincare method can be used only for $\varepsilon a^{2}<1$ and the second approximation is more accurate than the first one. For large $\varepsilon a^{2}(>1)$, the value of $T_{1}$ is no longer acceptable and that of $T_{2}$ becomes "irration". 
§3. Period from a modified Poincaré method

In this section, a modified Poincaré method is proposed.

Assuming that the strongly non - linear Duffing oscillator is near to certain weakly non - linear one with unknown frequency $\omega$, we rearrange the governing differential equation (1.1) as

$$
\ddot{x}+\omega^{2} x=\mu\left\{\left(\omega^{2}-1\right) x-\varepsilon x^{3}\right\},
$$

where $\mu$ is a formal parameter which is assumed to be small ( $\varepsilon$ plays now the role of an "ordinary" parameter).

The left hand side $\left(\ddot{x}+\omega^{2} x\right)$ represents a linear oscillator associated with the original strongly non-linear one; the right hand side $\left\{\left(\omega^{2}-1\right) x-\varepsilon x^{3}\right\}$ represents weak perturbations which consists of the linear perturbation $\left(\omega^{2}-1\right) x$ and the cubic ones $\left(-\varepsilon x^{3}\right)$.

As in the standard Poincaré method, the new time $\tau=\omega t$ is introduced and the equation (3.1) is rewritten as

$$
\omega^{2}\left(x^{\prime \prime}+x\right)=\mu\left\{\left(\omega^{2}-1\right) x-\varepsilon x^{3}\right\} .
$$

Then, both unknowns $x$ and $\omega$ are expanded in powers of $\mu$, that is

$$
\begin{aligned}
& x=x_{0}+\mu x_{1}+\mu^{2} x_{2}+\ldots \\
& \omega=\omega_{0}+\mu \omega_{1}+\mu^{2} \omega_{2}+\ldots,
\end{aligned}
$$

where $\omega_{0}$ is the "initial approximation" of the unknown frequency. Substituting the expansions (3.3), (3.4) into the equation (3.2) then equating coefficients of like powers of $\mu$ yield

$$
\begin{aligned}
\omega_{0}^{2}\left(x_{0}^{\prime \prime}+x_{0}\right) & =0 \\
\omega_{0}^{2}\left(x_{1}^{\prime \prime}+x_{1}\right) & =\left(\omega_{0}^{2}-1\right) x_{0}-\varepsilon x_{0}^{3} \\
\omega_{0}^{2}\left(x_{2}^{\prime \prime}+x_{2}\right) & =-2 \omega_{0} \omega_{1}\left(x_{1}^{\prime \prime}+x_{1}\right)+\left(\omega_{0}^{2}-1\right) x_{1}+2 \omega_{0} \omega_{1} x_{0}-3 \varepsilon x_{0}^{2} x_{1}, \\
\ldots \ldots & \ldots \ldots \ldots
\end{aligned}
$$

The general solution of the equation (3.5), satisfying the initial conditions (2.4) is

$$
x_{0}=a \cos \tau \text {. }
$$

Using (3.8), the equation (3.6) becomes

$$
\omega_{0}^{2}\left(x_{1}^{\prime \prime}+x_{1}\right)=\left[\left(\omega_{0}^{2}-1\right)-\frac{3}{4} \varepsilon a^{2}\right] a \cos \tau-\frac{\varepsilon a^{3}}{4} \cos 3 \tau .
$$

To eliminate the secular term, $\omega_{0}^{2}$ should be taken as

$$
\omega_{0}^{2}=1+\frac{3}{4} \varepsilon a^{2}
$$


Then, the solution $x_{1}$ satisfying the initial conditions (2.5) is:

$$
x_{1}=\frac{\varepsilon a^{3}}{32 \omega_{0}^{2}}(\cos 3 \tau-\cos \tau)
$$

With regard to (3.8), (3.10), (3.11), we rewrite the equation (3.7) in the form

$$
\omega_{0}^{2}\left(x_{2}^{\prime \prime}+x_{2}\right)=\left(2 \omega_{0} \omega_{1}+\frac{3 \varepsilon^{2} a^{4}}{128 \omega_{0}^{2}}\right) a \cos \tau+\frac{\omega_{1} \varepsilon a^{3}}{2 \omega_{0}} \cos 3 \tau-\frac{3 \varepsilon^{2} a^{5}}{128 \omega_{0}^{2}} \cos 5 \tau
$$

Eliminating the secular term gives:

$$
\omega_{1}=-\frac{3 \varepsilon^{2} a^{4}}{256 \omega_{0}^{3}}
$$

and the equation for determining $x_{2}$ takes the form:

$$
\omega_{0}^{2}\left(x_{2}^{\prime \prime}+x_{2}\right)=\frac{-3 \varepsilon^{3} a^{4}}{512 \omega_{0}^{4}} \cos 3 \tau-\frac{3 \varepsilon^{2} a^{5}}{128 \omega_{0}^{2}} \cos 5 \tau .
$$

The solution $x_{2}$ satisfying the initial conditions (2.5) is:

$$
x_{2}=\frac{3 \varepsilon^{3} a^{7}}{4096 \omega_{0}^{6}}(\cos 3 \tau-\cos \tau)+\frac{3 \varepsilon^{2} a^{5}}{3072 \omega^{4}}(\cos 5 \tau-\cos \tau) .
$$

Hence, the expansions of the frequency $\omega$, the period $T$ and the solution $x$ are respectively

- in the first approximation

$$
\begin{aligned}
& \omega_{*}^{(1)}=\omega_{0}=\sqrt{1+\frac{3}{4} \varepsilon a^{2},} \\
& T_{*}^{(1)}=\frac{2 \pi}{\omega_{0}}=\frac{2 \pi}{\sqrt{1+\frac{3}{4} \varepsilon a^{2}}}, \\
& x_{*}^{(1)}=a \cos \tau+\frac{\varepsilon a^{3}}{32 \omega_{0}^{2}}(\cos 3 \tau-\cos \tau) .
\end{aligned}
$$

- in the second approximation

$$
\begin{aligned}
\omega_{*}^{(2)}= & \omega_{0}+\omega_{1}=\sqrt{1+\frac{3}{4} \varepsilon a^{2}}-\frac{3 \varepsilon^{2} a^{4}}{256 \omega_{0}^{3}}, \\
T_{*}^{(2)}= & \frac{2 \pi}{\omega_{0}+\omega_{1}}=\frac{2 \pi}{\sqrt{1+\frac{3}{4} \varepsilon a^{2}}-\frac{3 \varepsilon^{2} a^{4}}{256 \omega_{0}^{2}}} \\
x_{*}^{(2)}= & a \cos \tau+\frac{\varepsilon a^{3}}{32 \omega_{0}^{2}}(\cos 3 \tau-\cos \tau)+\frac{3 \varepsilon^{3} a^{7}}{4097 \omega_{0}^{6}}(\cos 3 \tau-\cos \tau) \\
& +\frac{3 \varepsilon^{2} a_{*}^{5}}{3072 \omega_{0}^{4}}(\cos 5 \tau-\cos \tau) .
\end{aligned}
$$


The formulas in the third approximation are enough complicated

$$
\begin{aligned}
\omega^{(3)}= & \omega_{0}+\omega_{1}+\omega_{2}=\sqrt{1+\frac{3}{4} \varepsilon a^{2}}-\frac{3 \varepsilon a^{4}}{256 \omega_{0}^{3}}-\frac{45 \varepsilon^{4} a^{8}}{131.072 \omega_{0}^{4}} \\
T_{*}^{(3)}= & \frac{2 \pi}{\sqrt{1+\frac{3}{4} \varepsilon a^{2}}-\frac{3 \varepsilon^{2} a^{4}}{256 \omega_{0}^{3}}-\frac{45 \varepsilon^{3} a^{8}}{131.072 \omega_{0}^{7}}} \\
x_{*}^{(3)}= & \left(\frac{9 \varepsilon^{5} a^{11}}{262.144 \omega_{0}^{10}}-\frac{3 \varepsilon^{3} a^{7}}{16.384 \omega_{0}^{6}}\right)(\cos 3 \tau-\cos \tau)+\frac{9 \varepsilon^{4} a^{9}}{196.608 \omega_{0}^{8}}(\cos 5 \tau-\cos \tau) \\
& +\frac{9 \varepsilon^{3} a^{7}}{294.912 \omega_{0}^{6}}(\cos 7 \tau-\cos \tau) .
\end{aligned}
$$

The values of $T_{*}^{(1)}, T_{*}^{(2)}, T_{*}^{(3)}$ are shown in the fifth, sixth and seventh columns of the table.

We see that the results obtained from the method proposed are fully acceptable even for large values of $\varepsilon a^{2}$.

For $\varepsilon a^{2}=1000$, the relative errors of $T_{*}^{(1)}, T_{*}^{(2)}, T_{*}^{(3)}$ are respectively of orders $0.022,0.0008,0.00008$

\begin{tabular}{cccccccc}
\hline$\varepsilon a^{2}$ & & $T_{e x}$ & $T_{1}$ & $T_{2}$ & $T_{*}^{(1)}$ & $T_{*}^{(2)}$ & $T_{*}^{(3)}$ \\
\cline { 1 - 4 } 0. & $\overline{6}$ & $\overline{2} 28319$ & 6.28319 & 6.18319 & 6.28319 & 6.28319 & 6.28319 \\
0.01 & 6.25976 & 6.25971 & 6.25976 & 6.25976 & 6.25976 & 6.25976 \\
0.1 & 6.06066 & 6.05608 & 6.06087 & 6.06004 & 6.06066 & 6.06066 \\
1.0 & 4.76802 & 4.65959 & 4.85950 & 4.74964 & 4.76789 & 4.76802 \\
4. & 3.17971 & 2.51327 & 5.29110 & 3.14159 & 3.17884 & 3.17975 \\
10. & 2.19179 & 1.32278 & -1.81956 & 2.15511 & 2.19065 & 2.19187 \\
40. & 1.15182 & 0.39270 & -0.05452 & 1.12849 & 1.15095 & 1.15190 \\
100. & 0.73626 & 0.16320 & -0.00804 & 0.72073 & 0.73566 & 0.73632 \\
1000. & 0.23434 & 0.01671 & -0.00008 & 0.22928 & 0.23414 & 0.23436 \\
\hline
\end{tabular}

\section{Conclusions}

A modified Poincaré method is proposed. It consists in introducing a formal parameter in order to separate "weak perturbations" from the "associated linear oscillator" (the one which is near as possible of the strongly non-linear oscillator). Applying to an undamped Duffing oscillator having large cubic non-linearity, free oscillation periods computed are in good agreement with those obtained from the exact solution. 
Numerical computations in the present paper are performed by D. Tran Duong Tri

This publication is completed with financial support from The Council for Natural Science of Vietnam.

\section{REFERENCES}

1. Nayfeh A. N. Perturbation method Wiley, New York, 1973.

2. Cheung Y. K., Chen S. H. and Lan S. L. A modified Lindstedt - Poincaré method for certain strongly non-linear oscillators, Int. J. Non-linear Mechanics, Vol. 26, No $3 / 4$, pp, 367-378, 1991.

Received September 10, 2001

in revised form October 3, 2002

PHƯƠNG PHÁP POINCARÉ CHO CHẤN TƯ DUFFING PHI TUYẼ́N MẠNH

Một biến thể của phương pháp Poincaré được trình bày. Một tham số hình thức dược đưa vào để biến đổi chấn tử Duffing phi tuyến mạnh thành một chấn tử phi tuyến yếu. Tần số tính được rất sát với tần số chính xác kể cả khi có phi tuyến mạnh. 